\title{
Changing for the Better: Academic Scholars Find New Audience through Video Abstracts
}

\author{
Darshana T. Shah, PhD ${ }^{1}$
}

ABSTRACT

Author affiliations are listed at the end of this article.

The fallout from COVID-19 may fundamentally change the way we work over the next five years. Scholarly publishing is not exempt from COVID-19's impact.

Correspondence to: Darshana T. Shah, PhD Marshall University Joan C. Edwards School of Medicine shah@marshall.edu

\section{KEYWORDS}

Video Abstract

The COVID-19 pandemic has overturned nearly every aspect of life as we know it, from how we live and work, to how we interact with our students, patients, and other scholars in academic medicine. The fallout from COVID-19 may fundamentally change the way we work over the next five years. Scholarly publishing is not exempt from COVID-19's impact. Although change in academic publishing is constant, as evidenced by the recent transition to digital-first content and the acceleration of open-access and open-data through Plan $S^{1}$, the community is now confronting new challenges in the face of substantial unknown. Fortunately, advances in digital media technologies and the increased ubiquity of online media content have presented unprecedented opportunities for scholars to share their work through new genres and mediums of communication.

Beginning this issue, the Marshall Journal of Medicine is offering authors the option to submit a Video Abstract summarizing their work. The new feature is aimed at increasing the visibility of our authors and their work. A video abstract is an accompanying feature that attracts viewers to a published article such as the one published in this issue of MJM. Through streaming video media, authors can now expand the reach of published articles and provide a new, enhanced user experience for the Journal's global audience. Video abstracts can significantly expand an audience beyond fellow scientists, and as we know, greater visibility leads to greater citation.

Video not only provides an interactive, more engaging, and memorable experience for a reader, but also sparks curiosity about the topic and possibly encourages the viewer to learn and read more about it. Full details about this new feature can be found in the MJM instructions to authors. On behalf of the MJM editorial board, I encourage authors to submit a video abstract along with their articles and take advantage of this newly added feature.

\section{AUTHOR AFFILIATIONS}

1. Marshall University Joan C. Edwards School of Medicine, Huntington, West Virginia

\section{REFERENCE}

1. Stern BM, O'Shea EK. A proposal for the future of scientific publishing in the life sciences. PLoS Biology. 2019;17(2):e3000116. 